\title{
The virtual haptic display: A device for exploring 2-D virtual shapes in the tactile modality
}

\author{
JASON S. CHAN \\ Trinity College, Dublin, Ireland \\ Thorsten Maucher and Johannes Schemmel \\ University of Heidelberg, Heidelberg, Germany \\ Dana Kilroy and Fiona N. Newell \\ Trinity College, Dublin, Ireland \\ AND \\ KARLHEINZ MEIER \\ University of Heidelberg, Heidelberg, Germany
}

\begin{abstract}
In order to understand better the processes involved in the perception of shape through touch, some element of control is required over the nature of the shape presented to the hand and the presentation timing. To that end, we have developed a cost-effective, computer-controlled apparatus for presenting haptic stimuli using active touch, known as a virtual haptic display (VHD). The operational principle behind this device is that it translates black and white visual images into topographic, 2-D taxel (tactile pixel) arrays, along the same principle using in Braille letters. These taxels are either elevated or depressed at any one time representing white and black pixel colors of the visual image, respectively. To feel the taxels, the participant places their fingers onto a carriage which can be moved over the surface of the device to reveal a virtual shape. We conducted two experiments and the results show that untrained participants are able to recognize different, simple and complex, shapes using this apparatus. The VHD apparatus is therefore ideal at presenting 2-D shapes through touch alone. Moreover, this device and its supporting software can also be used for presenting computer-controlled stimuli in cross-modal experiments.
\end{abstract}

Generating tactile patterns over a rather large geometrical surface is a challenge in terms of mechanical design, electrical power consumption and cost. High-resolution 2-D tactile shapes can be reproduced using embossing paper (e.g., Lederman, Browse, \& Klatzky, 1988) and 3-D objects can be constructed using clay or other material such as Lego bricks (e.g., Newell, Ernst, Tjan, \& Bülthoff, 2001). Although these types of stimuli are adequate for haptic experimentation, it is nevertheless difficult to control the shape dimensions precisely or to maintain accurate timing using such stimuli. Furthermore, the development of more real-world paradigms, such as multisensory perception (e.g., presenting stimuli from more than one sensory modality), is compromised. Given the increase in the number of investigations on haptic and multisensory perception there is a real need for an experimental apparatus that is capable of delivering haptic stimuli under controlled spatial and temporal conditions. Moreover, such a device should be able to present visual as well as haptic stimuli under the same conditions.
There are, to date, several devices available on the market which are capable of presenting tactile displays. However, most of these devices have limitations. For example, many of the earlier computer controlled tactile simulators were stationary and only capable of delivering texture information of very small objects. Perhaps one of the better known of these virtual tactile displays was the Optacon, developed by Linvill and Bliss (1966). The Optacon involves a handheld optical scanner that translates luminance contrasts into a $6 \times 24$ matrix of vibratory pins. The Optacon is not a haptic system in the sense that it does not allow for active scanning of the stimulus. The matrix of tactile pins is immobile and the user must move the scanner (i.e., not the matrix of pins) across the surface with one hand while feeling what is on the virtual surface with the other hand. It is known that active touch provides better discrimination of objects (Klatzky \& Lederman, 1993; Lederman \& Klatzky, 1993; Lederman, Klatzky, \& Reed, 1993) and textures (Lederman, 1981) than passive touch. Consequently, the use of this type of apparatus

J.S.Chan, jason.chan@tcd.ie 
is not ideal for texture discrimination or shape tasks that require active touch.

Following along the lines of Linvill and Bliss, an apparatus developed by Ikei, Wakamatsu, and Fukuda (1997) called the vibratory tactile system, is able to simulate textures by translating visual textures into a $5 \times 10$ matrix of piano wires, driven by a series of vibrating piezoelectric actuators. While this apparatus is capable of simulating very complex textures, it has a very small perceptual "window" of a single fingertip. Moreover, the user must move the entire apparatus to feel larger textures. Given that this apparatus is rather large and heavy, an increase in friction is caused by an increase in object size which, in turn, can affect scanning characteristics.

Later devices allowed for the presentation of more complex stimuli such as Asian characters or letters (e.g., Saida, Shimizu, \& Wake, 1982; Yanagida, Kikita, Lindeman, Kume, \& Tetsutani, 2004). Both the Saida et al. and the Yanagida et al. devices have a large surface area (covering the abdomen or back, respectively) and allow for static presentation with relatively low resolution (Saida et al.'s devices comprises a $10 \times 10$ matrix array of actuators and Yanagida et al.'s device comprises a $3 \times 3$ matrix). In spite of the fact that active touch is not possible with these devices, the authors reported relatively high accuracy rates when active touch was simulated. For example, letter recognition accuracy rates increased to about $95 \%$ correct when the letter was presented as if it was being written on the participant but rates dropped to between $20 \%$ and $40 \%$ when the entire letter was presented as a whole.

Perhaps the best-known tactile virtual display is the tactile visual substitution system (TVSS) developed by Bach-y-Rita, Collins, Saunders, White, and Scadden (1969). This TVSS can be placed on the abdomen, back, thigh or even the finger and is connected to a portable camera which can also be operated by the user. By moving the camera, participants were able to perceive a 2-D representation of the visual shapes using the TVSS. This is done by translating digitized images (from the camera) to the matrix of tactile pins that is strapped to the participant's body area. Bach-y-Rita et al. (1969) reported that trained blind participants were able to accurately recognize objects and even complex objects such as faces.

More recently, Bach-y-Rita and his colleagues have further developed the TVSS to create the tongue display unit, or TDU (Bach-y-Rita, Kaczmarek, \& Meier, 1998; Bachy-Rita, Kaczmarek, Tyler, \& Garcia-Lara, 1998). The TDU is composed of 144 gold-plated copper electrodes, arranged in a $12 \times 12$ taxel matrix. The tongue does not have the protective layer of skin and is thus better able to perceive stimulation than the skin. Bach-y-Rita et al. have demonstrated that participants using this apparatus are capable of accurately perceiving shapes. A practical use for the TDU is for the training of patients with vestibular damage who have difficulty balancing their bodies in the absence of visual feedback (see Bach-y-Rita, Danilov, Tyler, \& Grimm, 2005, for an example).

While the perception of shapes using the TDU is very accurate, it can only present shapes that are no bigger than the size of the tongue without incurring image scaling. ${ }^{1}$
This tongue system also does not rely on active haptic scanning, known to facilitate object recognition (Gibson, 1962). Furthermore, the TDU has limited wearability since it is rather invasive and some participants find it uncomfortable to use.

Nowadays, a more ubiquitous virtual tactile display used in tactile perception research is a device known as a PHANTOM. PHANToM devices are capable of delivering virtual 2-D and 3-D objects in a highly controlled manner and have been used successfully to study human perception (see, e.g., Ernst \& Banks, 2002). These instruments use force-feedback to give the impression of an object in space. Haptic exploration with a PHANToM is limited to a single finger only and direct skin contact with the virtual object is not possible with this device.

While there are a number of tactile virtual devices available, these devices are limited either in the way in which stimuli are presented (e.g., single finger exploration as in the PHANToM), the type and size of the stimuli that can be presented, the portability of the device and the general cost. To address some of these limitations, we have developed an apparatus, which we call the virtual haptic display (VHD) that is both portable and capable of delivering simple shapes and scenes using active touch. Here, the user places their fingers onto a relatively small active area of 48 tactile elements (taxels) which are mounted onto a carriage. This carriage can then be moved by the user across a large surface area of the device $(165 \times 154 \mathrm{~mm})$ in order to reveal the virtual 2-D shape.

The primary advantages of our VHD compared to other virtual tactile displays are that the VHD allows for more ecologically valid exploration of a tactile shape or scene. In other words, several fingers can be used to explore a shape at any one time and hand movement is necessary to reveal shape information (see Technical Description). One other small but perhaps significant advantage of the VHD is that only the small active area requires mechanical elements, electrical power and computer control, thus minimizing power consumption and increasing portability.

The VHD is also capable of presenting visual stimuli simultaneously with the haptic stimuli. Furthermore, the visual and haptic stimuli can be congruent or incongruent in spatial characteristics depending on the requirements of the experimental design. For visual presentation of the stimuli, the VHD is versatile in that either an entire visual object can be presented or the object can be masked and viewed through a moveable aperture of varying size (see Technical Description). This feature may be important for the implementation of multisensory experiments in that it can allow experimenters to, for example, measure the contribution of each modality to the perception of an object.

Another feature of the VHD is its ability to record precisely the haptic scan paths made by the participant in each trial. Such scan path records are analogous to saccadic scans recorded by an eye tracker. Like eye movements, haptic scan path information can give an important insight into the perceptual and cognitive processes involved in shape perception (Yarbus, 1967). Furthermore, this capability can allow computer scientists to refine models of human multisensory object recognition that may sub- 
sequently be used in the development of artificial intelligence machines (e.g., Davison \& Frégnac, 2006)

\section{TECHNICAL DESCRIPTION OF VHD}

The VHD was developed as a single prototype with a cost of approximately $€ 2,000$ for materials. The authors have no financial interests. In the following section we describe the technical design and the dedicated software required to operate the VHD. The software allows for a fully automated generation of complex paradigms including a combination of tactile and visual displays presented under precise temporal and spatial control.

\section{Mechanical Description}

The technical drawing (Figure 1A) depicts the mechanical components of the VHD. An aluminium base holds two parallel linear guiding rails with a carriage moving on ball bearings (Type KUME 09 manufactured by INA Wälzlager, Herzogenaurach, Germany). A third identical rail is mounted perpendicular on the two parallel ones so that the carriage on this single horizontal rail can be moved with very little friction in the $x$ - and $y$-directions independently. The carriage holds the tactile output unit which consists of six standard piezoelectric Braille display elements. Each Braille element (METEC GmbH, Stuttgart, Germany) has eight independently movable plastic actuators arranged in

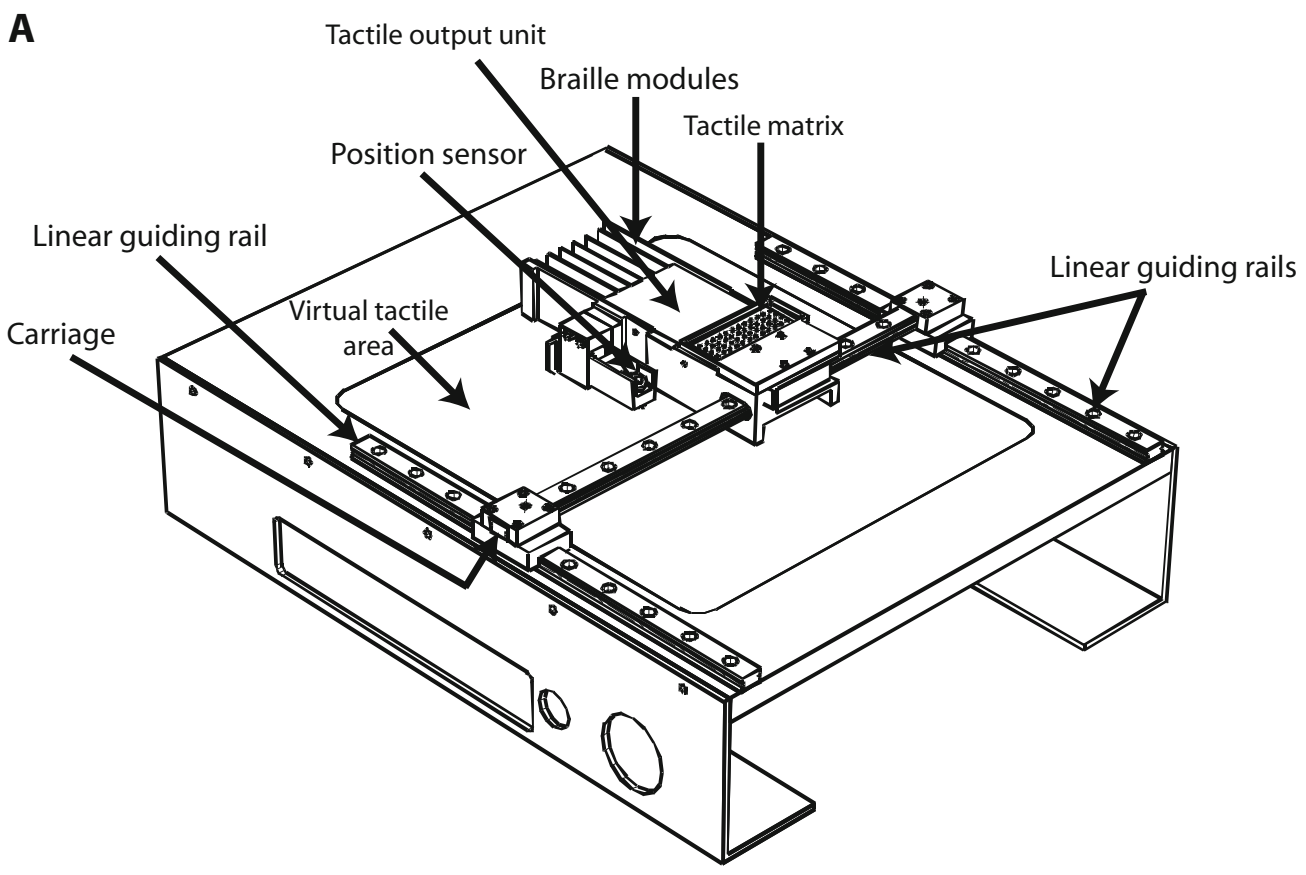

B

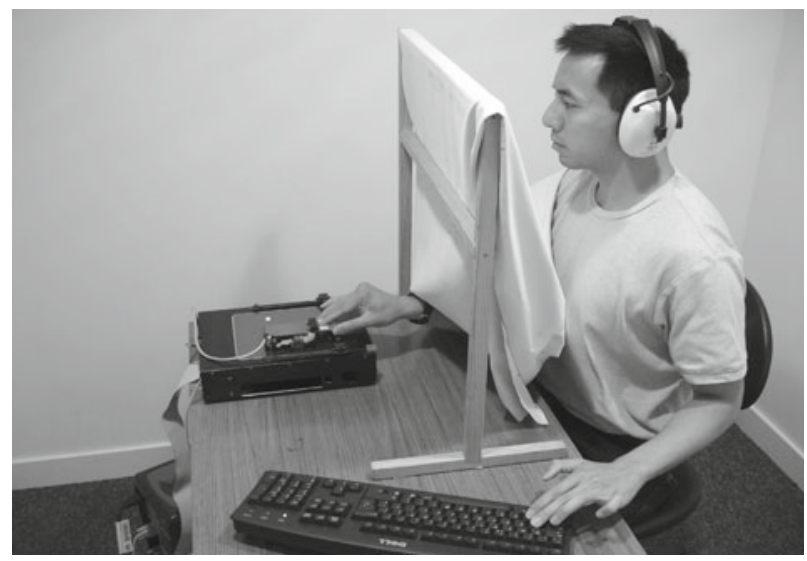

Figure 1. (A) Mechanical components of the VHD. The physical dimensions of the VHD are height $=$ $6.8 \mathrm{~cm}$, width $=23 \mathrm{~cm}$, and length $=30 \mathrm{~cm}$. The linear guide rails hold the carriage in the tactile area with little friction. The position sensor gives the host computer the coordinate data for accurately presenting the stimulus as well as for future scan path analysis. To feel the shape, the participant places their fingers onto the movable carriage which contains the tactile matrix. (B) Image of a participant using the VHD in a typical shape recognition experiment. Responses can be made via computer keyboard. Participants typically wear sound attenuating, circum-aural headphones to minimize any auditory cues produced by the taxels. 
a $2 \times 4$ matrix, allowing for a total matrix size of $12 \times 4$ taxels to be felt at any given moment. The tactile output unit can slide across an area of $165 \times 154 \mathrm{~mm}$ providing access to approximately 2,400 virtual taxels. Thus, the active surface of the tactile output unit (i.e., the area felt by the user) corresponds to only $2 \%$ of the fully accessible area at any given time.

An individual actuator pin is mounted on a pair of piezoelectric levers providing vertical movements of up to $0.7 \mathrm{~mm}$. The spacing between actuators is 3.21 and $2.45 \mathrm{~mm}$ in the $x$ - and $y$-directions, respectively. The tactile output unit consists of 48 actuators in total, distributed over an area of $43 \times 16 \mathrm{~mm}$, which can be comfortably covered by three fingertips.

The spatial position of each taxel is recorded by an optical sensor (similar to a computer mouse; Mouse Systems, Fremont, CA) located at the base of the carriage. A white reference point located at the upper right corner of the tactile area is detected by a reflective photo sensor and is used to define the "origin" of the coordinate system. This reference point is important while initializing the VHD since it tracks the exact location of the carriage in relation to the entire tactile area.

The VHD receives the pattern which corresponds to the position of the tactile matrix in respect to the image being displayed. The patterns are sent via a standard 24bit digital I/O PCMCIA card (DIO-24 manufactured by National Instruments) from the computer to the VHD. The computer receives the position data as well as the state of the calibrating sensor via the serial port. The position data are of twofold importance for the VHD. They provide information to the computer to calculate the correct pattern to be displayed on the tactile matrix and this position data can also be used to examine the user's scan path of exploration. The Braille elements need 200 Volts for the operation of the piezoelectric crystals which is generated locally in the VHD with a DC/DC converter from the $5 \mathrm{~V}$ available on the IO-Card.

\section{Description of the VHD Dedicated Software}

The VHD is programmed in $\mathrm{C}++$ with a Qt API add-in for the graphical user interface (Trolltech, Palo Alto, CA). This software environment provides a portable code in a standardized programming environment.

This dedicated software allows for images to be sent to the VHD in two different user-interface modes. In the drawing mode, points and lines can be drawn using a mouse on a visual display and are then presented instantly as virtual stimuli on the VHD. In the image mode, black and white image files of standard image formats (e.g., *.png, *.jpg, and *.gif) can be read into the VHD and displayed. For images with a larger size than the full tactile area of the VHD, the user can select a subsection of the image to be presented.

To display texture information, the texture density is derived from the grayscale images. There are two modes of encoding such gray scale images. In the binary mode, all pixels in an image above a fixed threshold in terms of gray levels are set to white so that the corresponding taxels are elevated. In contrast, pixels below the gray level threshold are coded as black and the corresponding taxels are depressed. The second mode of displaying gray scale images is by probability encoding. When using the probability encoding mode, 255 gray levels $(g)$ of an image are interpreted as probabilities proportional to $1 / g$. The update rate of the corresponding taxel is then proportional to this probability.

One important aspect of computer controlled stimuli is automated time sequence control. In other words, there is precise control over stimuli presentation within each trial. This can be done by the generation of paradigm files. Such an instruction-based paradigm file can also control the presentation of visual images on a computer screen. This added versatility allows for a visual stimulus to be presented alone or in combination with a haptic stimulus. In this case, visual stimuli must be created using an external image graphics program. Moreover, visual stimuli do not have to be the same as the haptic stimuli. This may be an important asset when running multisensory experiments.

The software allows for two different types of visual presentation. The first shows the entire image, regardless of pixel resolution. In other words, the resolution of the visual stimuli is not fixed to the resolution of the VHD. The second type is by presenting a visual "window." The aspect ratio of the visual window is resized to fit the aspect ratio of the VHD, and only a small portion of the image is shown at any given time (see Figure 2). The portion of the image that is visible can be moved in spatial correspondence with the carriage of the VHD. As in the first type of visual presentation, the visual resolution need not be the same as the resolution of the VHD.

\section{TACTILE SHAPE RECOGNITION EXPERIMENTS}

To test the efficiency of the VHD in delivering perceivable shapes, we conducted two experiments; one on the recognition of simple, 2-D shapes as a function of practice
A

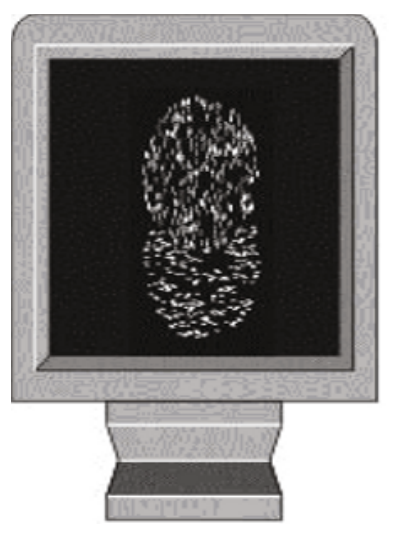

B

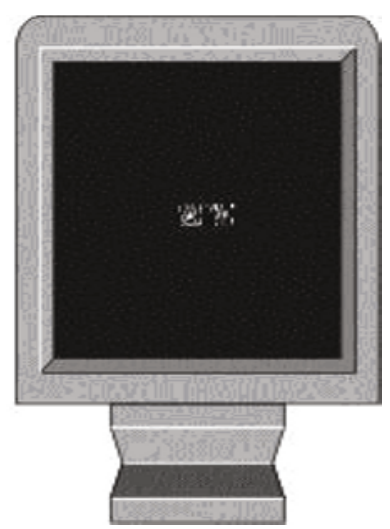

Figure 2. An example of the visual stimuli the VHD is capable of presenting. (A) The VHD is capable of presenting an entire visual stimulus while the haptic stimulus is also presented, or (B) a small visual "window" which is spatially correlated with the position of the VHD carriage can be presented. 
with the VHD and the second on the recognition of more complex tactile shapes (i.e., letters).

\section{EXPERIMENT 1}

In this experiment, we were interested in determining whether or not simple shapes could be perceived by the user and whether or not extensive training was required with the VHD for efficient perception. The participant's task was to explore the virtual shape and report its identity as fast and as accurately as possible.

\section{Method}

\section{Participants}

Ten postgraduate students from the Institute of Neuroscience at Trinity College ( 5 males and 5 females, between the ages of 19 and 29 years) agreed to participate in this task. Nine of the participants were right hand dominant. Six participants had no prior experience with the VHD and the remaining four had extensive practice with the VHD in other unrelated studies. None had previous practice with the stimuli used in the experiment. All participants reported to have no tactile impairments.

\section{Materials and Apparatus}

To test the limits of the apparatus we reduced the total number of taxels available to touch on the carriage. A range of taxels on the VHD carriage were covered with a small piece of cardboard, and a small hole revealing a $3 \times 3$ matrix of taxels $(7 \times 6 \mathrm{~mm})$ remained. The participant placed the index finger of their preferred hand over this matrix of taxels. This was also done to allow us to collect more accurate scan path data. It is unlikely that this reduced perceptual "window" significantly reduces shape recognition (Loomis, Klatzky, \& Lederman, 1991). The VHD was placed directly in front of the participant and behind a curtain. Participants were required to place their arm underneath the curtain to reach to the VHD (see Figure 1B). This ensured that visual information relating to limb position was not used as a cue to identify the objects. Participants also wore sound-attenuating, circum-aural headphones (Silencio, $\mathrm{NV}$ ) to minimize any possible auditory cues from movement of the VHD carriage and taxels.

The stimuli were composed of geometric 2-D shapes (square, vertical rectangle, isosceles triangle, and diamond). The areas of each shape were as follows: square $\left(100\right.$ taxels, or $\left.300 \mathrm{~mm}^{2}\right)$; rectangle (84 taxels, or $252 \mathrm{~mm}^{2}$ ); diamond (100 taxels, or $300 \mathrm{~mm}^{2}$ ), and triangle ( 84 taxels, or $126 \mathrm{~mm}^{2}$ ). The shapes were placed in the center of the tactile area (see Figure 3A). Each shape was presented 10 times during the course of the experiment. The VHD was connected to a Pentium 4 desktop computer which controlled stimulus presentation and recorded response times and scan paths. Trials were presented in a random order for each participant.

\section{Procedure}

Before the experiment began, participants were given the names of the four shapes they would feel and were asked to use these names when identifying the shapes. At the start of each trial, participants moved the carriage to the starting position, which was the far right corner of the virtual tactile area. When the trial began, participants were instructed to move the carriage in any direction to locate and explore the shape (i.e., scanning strategy was not restricted). Each shape was positioned in the center of the VHD. A trial lasted approximately 1 min or until a verbal response was made. At the end of the trial, the participant moved the carriage back to the starting position which triggered the offset of the stimulus. Participants' verbal responses and errors were recorded by the experimenter.

All participants performed 20 practice trials before the experimental blocks began. There was a total of 40 trials in the experimen- tal block. Feedback was not provided to the participants during the experimental blocks.

\section{Results and Discussion}

Figure 3B illustrates participants' accuracy performance on identifying each shape. Accuracy performance for both participant groups is above chance level for each of the shapes (i.e., 25\%). A mixed design ANOVA was conducted on the data with participant group as a between-groups factor (novices and experts) and shape as a within-subjects factor (square, rectangle, diamond, and triangle). There was a significant difference between experts and novices $[F(1,8)=93.73, p<.0001]$. Overall, the experts $(M=83.75 \%$ correct $)$ were significantly better than novices $(M=45.83 \%$ correct) in recognizing the shapes. There was no significant difference in recognition accuracy across the shapes $[F(1,8)<1$, n.s.].

In order to examine how much practice was required before accuracy performance was significantly improved, we compared performance to the novice group across trials as a function of the order of the trials. The forty trials were blocked into four according to the order of presentation, with Block 1 containing responses to the first 10 trials and

\section{A}
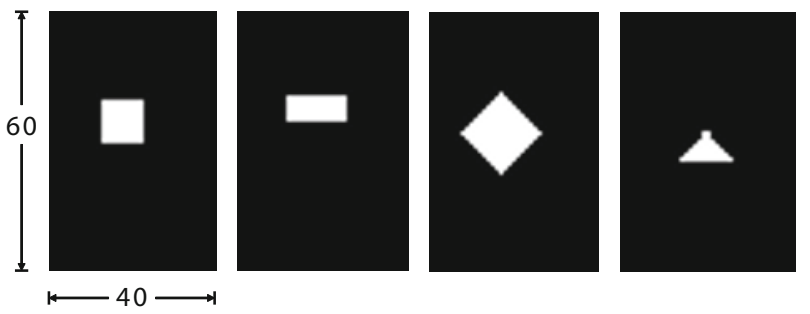

B

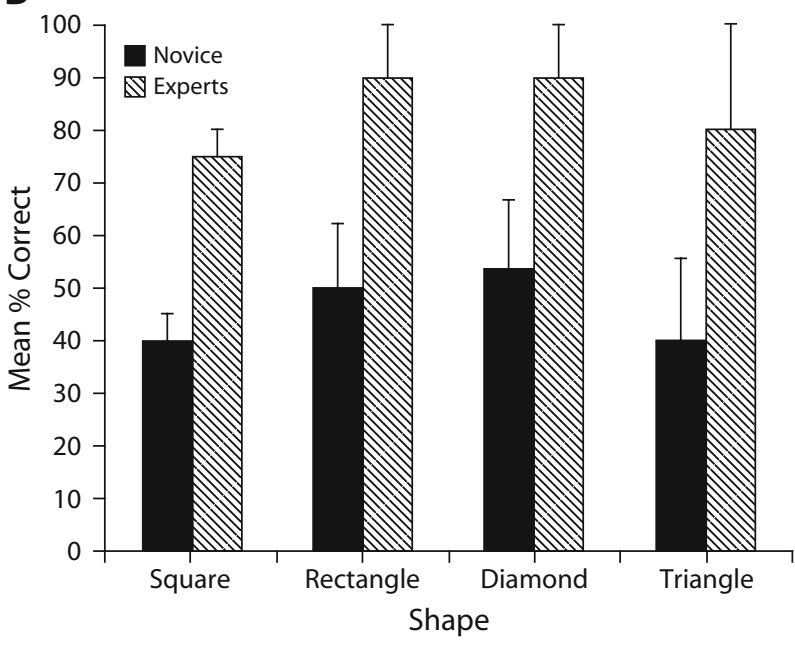

Figure 3. (A) A visual depiction of the four geometric shapes used in our shape recognition experiment. The shapes were presented as virtual tactile stimuli. The overall dimensions of the entire tactile area encompassing each shape are: 60 (length) $\times$ 40 (width) taxels. (B) Plot showing mean accuracy performance across each of the shapes for the novice and trained participant groups. The error bars represent \pm 1 standard error of the mean. 
Block 2 to the next 10, and so on. We conducted a one-way ANOVA with block order as the factor. We found no effect of block order $[F(3,20)<1$, n.s. $]$ indicating no increase in response accuracy across the blocked, 40 trials. It is clear, therefore, that practice of more than 40 trials is required before a response improvement is found in nonexperts but that, on the other hand, general practice with the device is sufficient to allow for relatively good performance across tasks (such as in our expert group).

\section{EXPERIMENT 2}

In the following experiment, we investigated whether complex shapes, which were familiar but not normally perceived through touch, could be recognized by a group of naive participants. To that end, we tested letter recognition through the VHD on participants who were not used to the device. Here the participant's task was to explore the virtual letter and report its identity as fast and as accurately as possible.

\section{Method}

\section{Participants}

Seventeen undergraduate and graduate students from Trinity College Dublin (10 males and 7 females, between the ages of 20 and 52 years) took part in this experiment. None took part in the previous experiment or had previous experience with the VHD. All participants were right-hand dominant and reported to have no tactile impairments.

\section{Materials and Apparatus}

The same apparatus used in Experiment 1 was also used here.

The stimuli in the experimental block were composed of eight letters which were chosen from the full alphabet to represent a good variation in shapes and features (A, C, D, H, J, K, M, and Z). These letters occupied a maximum area of $30 \mathrm{taxel}^{2}$ (i.e., about $100 \mathrm{~mm}^{2}$ ) on the surface of the VHD. The letters were presented in sans-serif font and were positioned in the center of the tactile area (see Figure 4A). Each shape was presented twice during the course of the experiment, in random order.

\section{Procedure}

Before the experiment began, participants were familiarized with the VHD and were given a maximum of 10 practice trials of tactile letter recognition using letter stimuli which were not used in the test (but occupying the same average space on the VHD as the experimental stimuli): L, E, P, M, and F. Before each practice trial, participants were given the name of five possible target letters and the participant was required to identify the correct target. Each practice letter was repeated twice in the practice block.

In the experimental block, participants were informed that they would be presented with target letters which may not necessarily include the practice letters and they should identify each as fast and as accurately as possible. They were also informed that letters could be repeated during the test block. They were not informed that the target stimuli were a restricted set of letters from the alphabet and, unlike in the practice block, were not informed of the possible targets before each trial.

As in the previous experiment, at the beginning of each trial, participants moved the carriage to the starting position, which was the far right corner of the virtual tactile area. When the trial began, participants were instructed to move the carriage in any direction to locate and explore the letter (i.e., scanning strategy was not restricted). There was no time limit on exploration. At the end of the trial, the participant moved the carriage back to the starting position which triggered the offset of the stimulus. Participants' verbal responses and errors were recorded by the experimenter. There were 16 trials in the experimental block (i.e., two repeats of each of the target letters).

\section{Results and Discussion}

Figure 4B illustrates participants' mean recognition performance in percentage correct for each of the target letters. A one-sample $t$ test was conducted examining performance between each letter and chance level (i.e., 1/26, or $3.85 \%)$. Accuracy performance for each letter was significantly higher than chance $(\mathrm{A}, t=3.49, p<.05 ; \mathrm{C}, t=$ $13.79, p<.0001 ; \mathrm{D}, t=2.70, p<.05 ; \mathrm{H}, t=7.10, p<$ $.0001 ; \mathrm{J}, t=19.13, p<.0001 ; \mathrm{K}, t=5.19, p<.0001 ; \mathrm{M}$, $t=7.32, p<.0001 ; \mathrm{Z}, t=5.90, p<.0001)$.

An item-analysis ANOVA across performance to the target letters revealed a significant difference in letter recognition $[F(1,16)=145.10, p<.0001]$. Participants were most accurate with the letters $\mathrm{C}$ and $\mathrm{J}$ relative to the letters $\mathrm{K}, \mathrm{A}$, and $\mathrm{D}(p<.05)$. Although performance was greater than chance level, participants had the most difficulty accurately recognizing the letter D and were more likely to respond $\mathrm{O}$ or $\mathrm{P}$ instead of the correct letter D. We surmised that given the similarity between these letters that $\mathrm{D}$ was often confused on the basis of the number of features in common with the incorrectly reported letters.

\section{A}

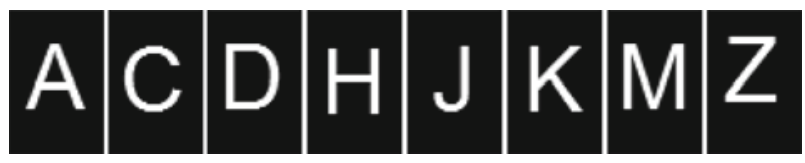

B

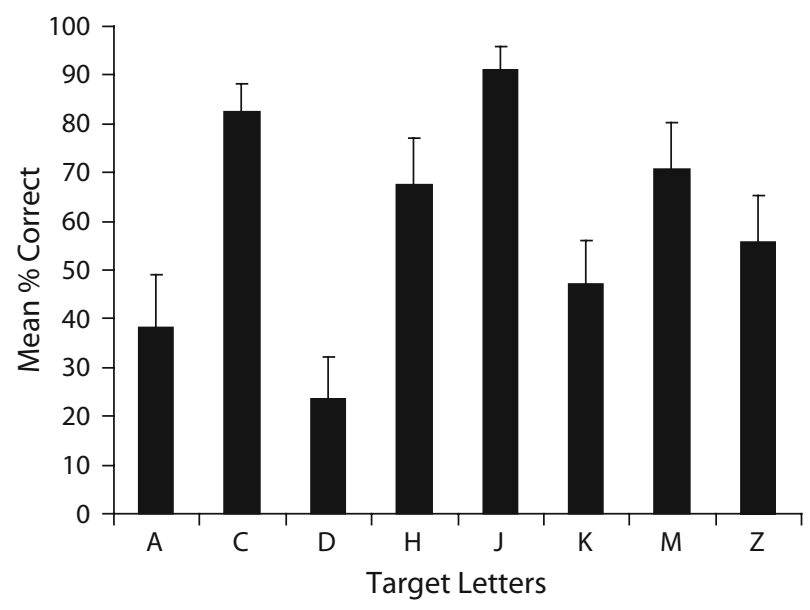

Figure 4. (A) A visual depiction of the eight letters used in the recognition experiment. The letters were presented as virtual tactile stimuli. The overall dimensions of the entire tactile area encompassing each shape are: 60 (length) $\times 40$ (width) taxels. (B) Plot showing mean accuracy performance across each of the target letters used in the task. In all cases, performance was above chance (i.e., 1/26). The error bars represent \pm 1 standard error of the mean. 


\section{TACTILE SCAN PATHS}

The VHD allows the experimenter to record the scan or exploration paths taken by the participant for each stimulus presentation. Figure 5 illustrates an example of a scan path taken from one participant in each experiment before a 2-D shape was recognized (A) or a target letter was identified (B). As can be seen from these example scan paths in (A), in the correctly identified shape trials this participant traced the contour of each shape as the shapes are easily resolvable from the scan paths. Furthermore, a similar contour-tracing strategy was also adopted by another participant in the letter recognition task (B) and in this example at least some of the target letters can be resolved from the scan paths, particularly the letters $\mathrm{C}, \mathrm{K}, \mathrm{Z}, \mathrm{A}$, and J. In fact, across all of the correct trials in Experiment 1, approximately $61.90 \%$ were trials in which the participant had adopted a contour tracing scan as opposed to $14.29 \%$ trials where a left/right or up/down sweeping exploration and $23.81 \%$ trials where a random scan was adopted. In the letter recognition task (Experiment 2), we found that in $83.83 \%$ of correct trials participants adopted a letter contour tracing scan whereas in $14.53 \%$ the scans were from left to right (or up-down) and in the remaining 1.64\% the stimuli were randomly explored. Thus, for both letters and simple 2-D stimuli contour tracing seemed to be the most effective strategy for correctly identifying the target, particularly so for the more complex letter stimuli.

Previous studies have suggested that exploration procedures in touch are contingent on the task (Klatzky \& Lederman, 1993; Lederman \& Klatzky, 1993; Lederman et al., 1993). In tactile shape identification, for example, contour following appears to be the most efficient exploration procedure (Klatzky \& Lederman, 1993; Lederman \& Klatzky, 1993; Lederman et al., 1993). In some of the incorrect trials (see bottom rows of Figure 5, panels A and B), the participant's scan path does not match the contour of the shape but instead follows a stereotyped, left-to-right exploration strategy (e.g., square and diamond exploration in panel A or most incorrect trials in panel B) or a random scanning (e.g., triangle in panel A). We found that a contour tracing scan path was mainly absent in the trials with an incorrect response (on average only $8.5 \%$ of incorrect trials consisted of contour tracing compared to about $84 \%$ of correct trials). This may suggest that contour tracing in the correct trials acts as a confirmation of the identity of the letter but that recognition may have occurred prior to the full tracing of the contour of the letter. The scan paths to the correct trials for both shapes and letters may suggest some sort of top-down processing whereby participants were trying to match what was being felt to a preconceived mental image (Sathian \& Zangaladze, 2002; Zhang, Weisser, Stilla, Prather, \& Sathian, 2004). In any case, these scan paths can reveal why the correct shape was not identified as well as indicate which features are the most informative for identifying each shape.

\section{GENERAL DISCUSSION}

In this article, we describe a virtual haptic device which is currently being used to present 2-D virtual images to the haptic modality. The VHD described here uses taxels on a small movable carriage to represent virtual shapes. This VHD is a versatile and compact apparatus which can present virtual haptic stimuli with a reasonable resolution. Although there are virtual haptic systems already available, those systems tend to be unwieldy, restrictive in terms of active exploration, invasive, or rather costly.

We tested the usability of this apparatus on a number of participants who were either required to perform a simple 2-D shape recognition task or a more complex letter recognition task. Although the spatial resolution of the VHD is not as high as other available devices (e.g., the apparatus developed by Ikei et al., 1997) the taxel resolution does seem to be more than adequate for the presentation of geometric shapes, letters and simple textures. With little training our participants were able to correctly identify simple shapes in Experiment 1 and identify letters in Experiment 2. Moreover, the VHD can capture exploration procedures or scan paths which can help the investigator better elucidate the processes involved in tactile shape perception. Participants with more practice using the VHD (i.e., experts in Experiment 1) were able to recognize the 2-D shapes with significantly greater accuracy. It is important to note that the group of expert participants have never performed an object recognition task using the VHD, but other unrelated psychophysical experiments. In other words, it is unlikely that their relatively better performance is due to their experience with other tactile object recognition studies using the VHD.

It is difficult to compare accuracy performance using the VHD with other devices since previous studies examining recognition of complex stimuli did not employ an active scanning method (see, e.g., Saida et al., 1982; Yanagida et al., 2004). However, when complex characters such as Japanese letters were presented sequentially (i.e., as if being written on the participant) accuracy performance was reported to be between $87 \%$ and $95 \%$ (in the Yanagida et al. and Saida et al. studies, respectively). Interestingly, performance among our expert participants is comparable to the performance in these studies but not to performance in our novice group. Thus, practice with the device has a clear effect on performance and in order to compare performance across devices, practice time needs to be controlled.

The VHD is a versatile apparatus in that it can also be used to translate visual text into taxels which can be a useful feature for the visually impaired since the taxels are placed in the standard Braille format. Thus, the VHD cannot only present shapes and standard letters but Braille letters as well. Previous studies investigating the scanning patterns of Braille readers have involved video recording the hand movements followed by subsequent visual examination of each video frame (Millar, 1984, 1997). Such studies have concluded that persons who are blind scan Braille letters with their hands in much the same way as the eyes of sighted persons scan visual text. However, these results are somewhat subjective since these data rely on the interpretation of the experimenter on the nature of the scan path. The VHD can provide a more objective and precise scanning record to further examine Braille reading in the visually impaired. 
A

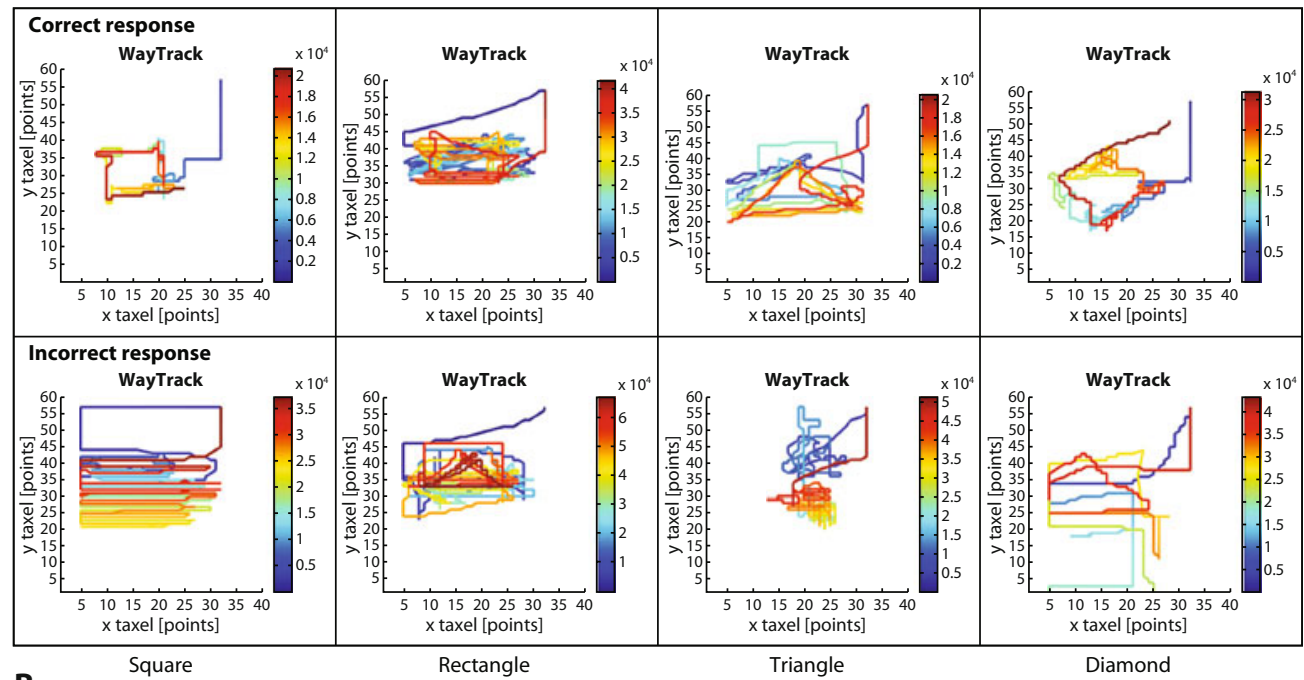

B
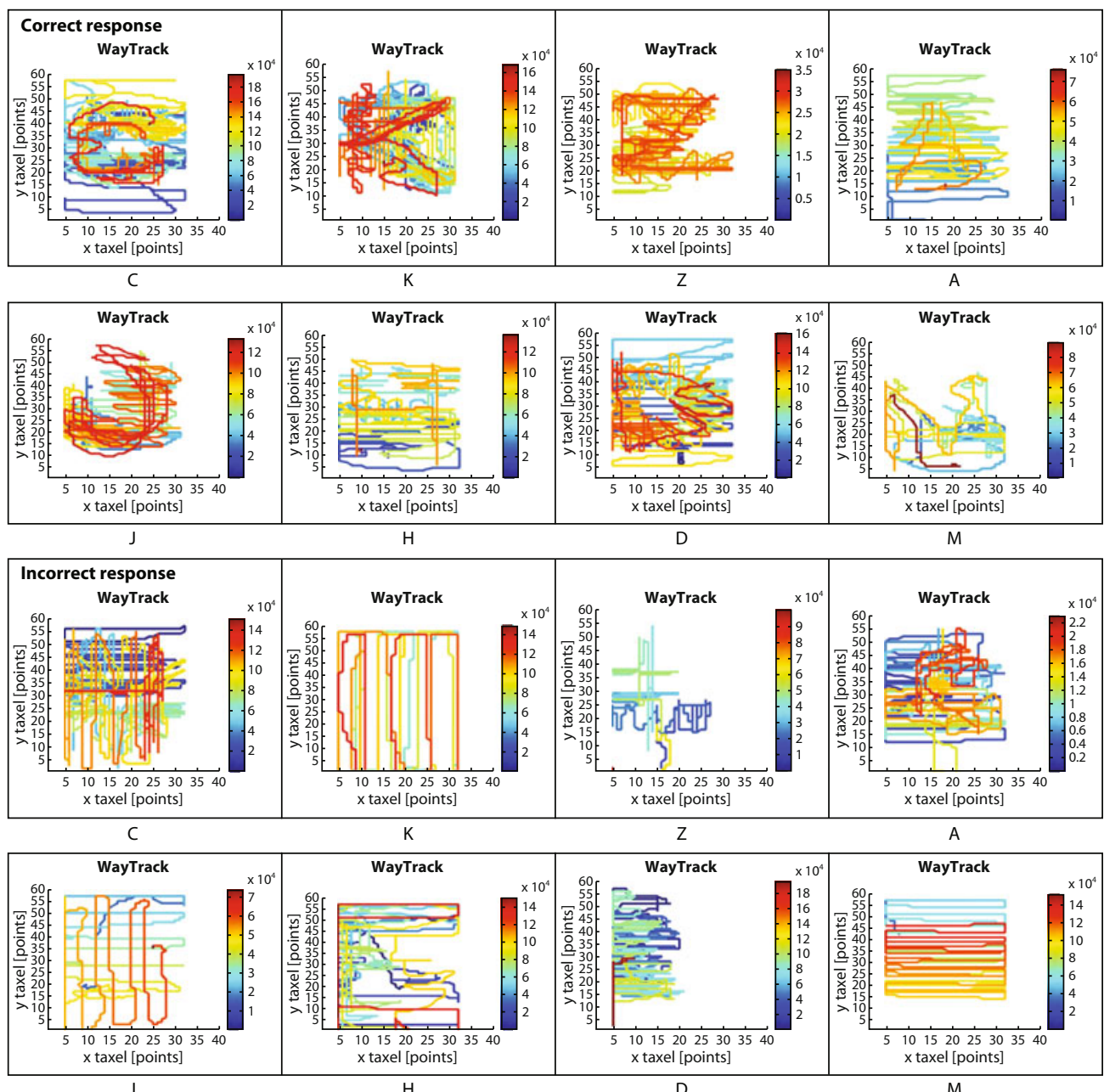

Figure 5. An example of the haptic scan paths (i.e., exploration procedures) created by participants during (A) shape exploration and (B) letter exploration. Each plot represents one trial for one participant. In panels $A$ and $B$, the upper row displays some of the scan paths when participants made a correct response, whereas the lower row represents the scan paths for incorrect responses to the same stimulus. Corresponding target stimuli are indicated below these rows. Elapsed time during the trial is color coded with blue representing the beginning of the scan sequence and red representing the end of the scan. 
The VHD's accompanying software also allows for the presentation of visual and tactile stimuli either simultaneously or in sequence under controlled temporal conditions. This aspect of the VHD is important for research into cross-modal shape identification. We are currently, and successfully, using the VHD to study multisensory shape and spatial perception (Chan \& Newell, 2007). The VHD is also capable of presenting a visual stimulus in its entirety or can be masked allowing the stimulus to be perceived through a virtual aperture, where only the area that is spatially correlated with the position of the VHD carriage is presented. It is important to remember that this visual stimulus need not be the same as the haptic stimulus, or of the same dimensions since images can be easily scaled. This may be a particularly useful feature when running multisensory experiments where intersensory conflict may be an important independent variable. The software will automatically rescale the visual window in proportion to the visual stimulus. It is also important to note that since the VHD is an integrated computer apparatus the timing between haptic, visual or even auditory stimuli can be easily coordinated.

In summary, we describe a versatile apparatus for presenting haptic stimuli that can be coordinated with the presentation of visual stimuli. We feel that the VHD provides a good compromise between cost, physical size, and spatial resolution. Furthermore, to the best of our knowledge, this is also the only apparatus available that can be used directly in visuo-haptic multisensory recognition experiments using relatively complex shapes.

\section{AUTHOR NOTE}

This research was funded by the European Union's Information System Technologies Programme Grant IST-2001-34712 and the Irish Research Council for the Humanities and Social Sciences. For a detailed schematic of this apparatus, please e-mail K. Meier at meierk@kip.uni-heidelberg .de. Correspondence concerning this article should be addressed to J. S. Chan, School of Psychology and Institute of Neuroscience, Trinity College, Dublin 2, Ireland (e-mail: jason.chan@tcd.ie).

\section{REFERENCES}

Bach-Y-Rita, P., Collins, C. C., Saunders, F. A., White, B., \& ScadDEN, L. (1969). Vision substitution by tactile image projection. $\mathrm{Na}$ ture, 221, 963-964.

Bach-Y-Rita, P., Danilov, Y., Tyler, M., \& Grimm, R. J. (2005). Late human brain plasticity: Vestibular substitution with a tongue BrainPort human-machine interface. Plasticidad y Restauración Neurológica, 4, 31-34.

Bach-Y-Rita, P., KaczmareK, K., \& Meier, K. (1998, October). The tongue as a man-machine interface: A wireless communication system. Paper presented at the 1998 International Symposium on Information Theory and Its Applications, Mexico City, Mexico.

Bach-Y-Rita, P., KaczmareK, K., Tyler, M., \& Garcia-Lara, J. (1998). Form perception with a 49-point electrotactile stimulus array on the tongue. Journal of Rehabilitation Research Development, 35, 427-430.
Chan, J. S., \& Newell, F. N. (2007). Measuring the distortion of haptic space using a virtual haptic device. Manuscript in preparation.

DAVISON, A., \& FRÉGNAC, Y. (2006). Learning cross-modal spatial transformations through spike timing-dependent plasticity. Journal of Neuroscience, 26, 5604-5615.

ERNST, M. O., \& BANKS, M. S. (2002). Humans integrate visual and haptic information in a statistical optimal fashion. Nature, 415, 429-433.

Gibson, J. J. (1962). Observations on active touch. Psychological Review, 69, 477-491.

IKeI, Y., WaKamatsu, K., \& FuKUda, S. (1997). Vibratory tactile display of image-based textures. Computer Graphics \& Applications, IEEE, 17, 53-61.

KLAtZKy, R. J., \& Lederman, S. J. (1993). Toward a computational model of constraint-driven exploration and haptic object identification. Perception, 22, 597-621.

Lederman, S. J. (1981). The perception of surface roughness by active and passive touch. Bulletin of the Psychonomic Society, 18, 253-255.

Lederman, S. J., Browse, R. A., \& Klatzky, R. J. (1988). Haptic processing of spatially distributed information. Perception \& Psychophysics, 44, 222-232.

Lederman, S. J., \& KlatZKY, R. J. (1993). Extracting object properties through haptic exploration. Acta Psychologica, 84, 29-40.

Lederman, S. J., Klatzky, R. J., \& Reed, C. L. (1993). Constraints on haptic integration of spatially shared object dimensions. Perception, 22, 723-743.

LinVILL, J., \& Bliss, J. (1966). A direct translation reading aid for the blind. Proceedings of the IEEE, 54, 40-51.

Loomis, J. M., Klatzky, R. L., \& Lederman, S. J. (1991). Similarity of tactual and visual picture recognition with limited field of view. Perception, 20, 167-177.

MillaR, S. (1984). Strategy choices by young Braille readers. Perception, 13, 567-579.

Millar, S. (1997). Reading by touch. London: Routledge.

Newell, F. N., Ernst, M. O., Tuan, B. S., \& BÜlthoff, H. H. (2001). Viewpoint dependence in visual and haptic object recognition. Psychological Science, 12, 37-42.

Saida, S., Shimizu, Y., \& WaKe, T. (1982). Computer-controlled TVSS and some characteristics of vibrotactile letter recognition. Perceptual \& Motor Skills, 55, 651-653.

Sathian, K., \& Zangaladze, A. (2002). Feeling with the mind's eye: contribution of visual cortex to tactile perception. Behavioural Brain Research, 135, 127-132.

Yanagida, Y., Kakita, M., Lindeman, R. W., Kume, Y., \& TetsuTANI, N. (2004, March). Vibrotactile letter reading using a lowresolution tactor array. Paper presented at the 12th International Symposium on Haptic Interfaces for Virtual Environment and Teleoperator Systems, Chicago.

Zhang, M., Weisser, V. D., Stilla, R., Prather, S. C., \& Sathian, K. (2004). Multisensory cortical processing of object shape and its relation to mental imagery. Cognitive, Affective, \& Behavioral Neuroscience, 4, 251-259.

\section{NOTE}

1. It could be argued that for visual perception the retina is also small and, hence, the size of the sense organ may not be an issue. However, unlike vision, touch perception relies on proximal or direct stimulation of the object onto the receptors therefore the size of the object that can be perceived is directly related to the size of the sense organ.

(Manuscript received November 28, 2005; revision accepted for publication December 27, 2006.) 